LA-14380

Approved for public release;

distribution is unlimited.

Nonstandard Jump Functions for

Radially Symmetric Shock Waves

- Los Alamos 
Edited by Mable Amador, Group IRM-CAS.

Photocomposition by Deidre' A. Plumlee, Group IRM-CAS.

Los Alamos National Laboratory, an affirmative action/ equal opportunity employer, is operated by Los Alamos National Security, LLC, for the National Nuclear Security Administration of the U.S. Department of Energy under contract DE-AC52-06NA25396.

This report was prepared as an account of work sponsored by an agency of the U.S. Government. Neither Los Alamos National Security, LLC, the U.S. Government nor any agency thereof, nor any of their employees make any warranty, express or implied, or assume any legal liability or responsibility for the accuracy, completeness, or usefulness of any information, apparatus, product, or process disclosed, or represent that its use would not infringe privately owned rights. Reference herein to any specific commercial product, process, or service by trade name, trademark, manufacturer, or otherwise does not necessarily constitute or imply its endorsement, recommendation, or favoring by Los Alamos National Security, LLC, the U.S. Government, or any agency thereof. The views and opinions of authors expressed herein do not necessarily state or reflect those of Los Alamos National Security, LLC, the U.S. Government, or any agency thereof. Los Alamos National Laboratory strongly supports academic freedom and a researcher's right to publish; as an institution, however, the Laboratory does not endorse the viewpoint of a publication or guarantee its technical correctness. 
LA-14380

Issued: October 2008

\section{Nonstandard Jump Functions for \\ Radially Symmetric Shock Waves}

Roy S. Baty

Don H. Tucker*

Dan Stanescu**

*University of Utah, Salt Lake City, Utah 84112-0090

**University of Wyoming, Laramie, Wyoming 82071

\section{- Los Alamos}





\title{
Nonstandard Jump Functions for Radially Symmetric Shock Waves
}

\author{
by
}

\author{
Roy S. Baty, Don H. Tucker, and Dan Stanescu
}

\begin{abstract}
Nonstandard analysis is applied to derive generalized jump functions for radially symmetric, one-dimensional, magnetogasdynamic shock waves. It is assumed that the shock wave jumps occur on infinitesimal intervals, and the jump functions for the physical parameters occur smoothly across these intervals. Locally integrable predistributions of the Heaviside function are used to model the flow variables across a shock wave. The equations of motion expressed in nonconservative form are then applied to derive unambiguous relationships between the jump functions for the physical parameters for two families of self-similar flows. It is shown that the microstructures for these families of radially symmetric, magnetogasdynamic shock waves coincide in a nonstandard sense for a specified density jump function.
\end{abstract}

\section{Introduction}

In this article, generalized jump conditions are derived using nonstandard analysis for radially symmetric, one-dimensional, diverging and converging shock waves. The shock waves are modeled using the magnetogasdynamic equations in nonconservative form, assuming two families of similarity variables that reduce the governing partial differential equations into ordinary differential equations (ODEs). Nonstandard predistributions of the Heaviside functions are applied to model the microstructure of a shock wave jump. The microstructure of a flow consists of the functions that represent the field variables such as density, velocity, pressure, and magnetic flux across a shock 
wave. A shock jump is modeled to occur over an infinitesimal interval with nonstandard jump functions. The nonstandard jump functions applied in the present analysis are given as follows: for a standard jump function $\phi(x)$, with jump $[\phi] \equiv \phi_{1}-\phi_{0}$ at $x=0$, define a nonstandard jump function by

$$
{ }^{*} \phi(x) \equiv \phi_{0}+[\phi]^{*} H(x)
$$

where ${ }^{*} H(x)$ is a nonstandard Heaviside function. Each flow parameter is assumed to have a distinct nonstandard jump function associated with it at the shock. This means that each flow variable may have a different nonstandard Heaviside function or microstructure across a shock wave. If the various Heaviside functions for the flow parameters have their jumps located on the same infinitesimal interval, then the governing equations, with products of generalized functions, yield unambiguous relationships between these Heaviside functions. Therefore, the use of predistributions of the Heaviside functions removes the mathematical inconsistencies associated with products of generalized functions such as $\delta \cdot H$ for the shock problems presented.

The present work generalizes the authors' research, Baty et al. [1, 2], on constructing nonstandard solutions across shock waves to radially symmetric, one-dimensional shock waves. The development shows that the microstructure for radially symmetric, magnetogasdynamic shock waves coincides and exhibits a weak uniqueness property (in a nonstandard sense that is made precise in the development) for all such flow fields.

\section{Equations of Motion}

\subsection{Radially Symmetric Magnetogasdynamics}

The equations governing the motion of symmetric, one-dimensional magnetogasdynamic (MGD) flows are given by

$$
\begin{aligned}
& (\ln \rho)_{t}+u(\ln \rho)_{r}+u_{r}+\frac{\kappa u}{r}=0, \\
& u_{t}+u u_{r}+\nu p_{r}+\mu \frac{\nu h}{r}(r h)_{r}=0, \\
& h_{t}+u h_{r}+h u_{r}+(\kappa-1) \frac{h u}{r}=0,
\end{aligned}
$$


and

$$
e_{t}+u e_{r}+\nu p\left(u_{r}+\frac{\kappa u}{r}\right)=0,
$$

where $\rho$ is the density, $\nu$ is the specific volume $\left(\equiv \frac{1}{\rho}\right), u$ is the velocity, $p$ is the pressure, $h$ is the tangential magnetic field strength, and $e$ is the specific energy. The notation for partial differentiation is simplified to the subscript notation $\xi_{r} \equiv \frac{\partial \xi}{\partial r}$. Here $\kappa=1,2$ represents cylindrical and spherical coordinate systems, respectively; and $\mu$ is the magnetic permeability. Equations (2) to (5) are discussed in Christer and Helliwell [3] for cylindrical flows, and in Srivastava [4] for spherical flows.

The conservation of mass, momentum, magnetic flux, and energy Equations (2), (3), (4), and (5), represent four equations in terms of five physical variables. To close the system of equations, two equations of state must be added. The equations of state relate the thermodynamic variables $\rho, p, e$, and $T$. Assuming a calorically perfect gas, the energy Equation (5) may be shown to be equivalent to a conservation equation written in terms of entropy. Because shock propagation is not an isentropic process, any conservation equation derived assuming Equation (5) and a perfect gas will not be satisfied across a shock wave; therefore, the Equations (2) to (5) are replaced by the under-determined system of Equations (2) to (4) together with an entropy condition defined behind a shock. Generalized solutions relating the jump functions for $\nu, u, p$, and $h$ are then determined by specifying one of the unknown flow parameters and integrating the resulting Equations (2) to (4) in terms of this field variable.

The fourth term in the momentum equation consists of a product of the specific volume and the magnetic field with its spatial derivative. Because such nonlinearities produce approximation specific results for generalized solutions, nonstandard techniques are applied to analyze the equations across shock wave discontinuities. Nonstandard analysis simplifies the analysis of the equations and allows the derivation of physically correct relationships between the generalized solutions for the shock wave jump functions. Because the governing equations of motion are inviscid, the resulting system of differential equations are underdetermined and cannot yield a unique theoretical or physical microstructure. 


\subsection{Similarity Variables}

The governing equations of motion are considered on two families of characteristic curves in the space-time domain. Along the characteristic curves, the equations of motion reduce to ordinary differential equations. The two families of characteristic curves correspond to diverging and converging MGD shock waves. Following the developments of Zel'dovich and Raizer [5], a similarity variable is introduced to define the characteristic curves:

$$
\xi=\frac{r}{R} \text { and } R=R(t)
$$

Two forms of the similarity variable are considered here:

$$
\xi_{i}=\frac{r_{i}}{R_{i}}=\frac{r_{i}}{A\left((-1)^{i+1} t_{i}\right)^{\alpha(i)}}
$$

where $i=1,2$ represents diverging and converging shock waves, respectively.

For both families of shock waves, the flow variables are assumed to be of the form

$$
p\left(\xi_{i}\right)=\rho_{0}\left(R_{i}\right) \dot{R}_{i}^{2} \pi\left(\xi_{i}\right), \rho\left(\xi_{i}\right)=\rho_{0}\left(R_{i}\right) g\left(\xi_{i}\right), u\left(\xi_{i}\right)=\dot{R}_{i} v\left(\xi_{i}\right),
$$

and

$$
h\left(\xi_{i}\right)=\sqrt{\rho_{0}\left(R_{i}\right)} \dot{R}_{i} \eta\left(\xi_{i}\right) .
$$

In Equations (6) to (9), $R_{i}$, the location of the shock wave, and $\rho_{0}$, the density in front of the shock wave, are the reference scales for the similarity solution; $A$ and $\alpha(i)$ are constants.

For diverging shock waves $(i=1)$, the variables of Equations (6) and (7) are defined on

$$
0 \leq t_{1}<\infty, r_{1} \leq R_{1}<\infty \text {, and } 0 \leq \xi_{1}<1
$$

and for converging shock waves $(i=2)$, the variables are defined on

$$
-\infty<t_{2} \leq 0, R_{2} \leq r_{2}<\infty, \text { and } 1 \leq \xi_{2}<\infty .
$$

The similarity variable of Equation (11) also represents diverging shock waves emerging from the surface of a star, Srivastava [4]. In all cases, the shock front is located at $\xi_{i}=1$ and the shock speeds are given by $D \equiv \dot{R}_{i}$.

Recalling that shock wave propagation is not an isentropic process, Equations (2), (3), and (4) are used to define ODEs relating the density $\rho$, velocity 
$u$, pressure $p$, and magnetic field $h$ across diverging shock waves. Because the resulting differential equations will be underdetermined, unique jump functions cannot be found using these equations. However, the analysis will yield unambiguous relationships between the jump functions for the flow parameters. A discussion of the uniqueness of the jump functions is given in Baty and Tucker [2].

\section{$3 \quad$ Nonstandard Generalized Jump Functions}

\subsection{Predistributions of Generalized Functions}

Nonstandard analysis studies the extension of number systems and function spaces to quotient spaces, which contain idealized elements that are infinitely large and infinitely small. For example, the real numbers, $\mathbb{R}$, may be extended to a hyperreal number system * $\mathbb{R}$ that is defined as a quotient space resulting from applying an equivalence relation $\sim$ on the set of sequences of real numbers, $\mathbb{R}_{s}$, indexed by the natural numbers $\mathbb{N}$ as follows

$$
{ }^{*} \mathbb{R} \equiv \mathbb{R}_{s} / \sim \text {. }
$$

The equivalence relation $\sim$ is defined by selecting an ultrafilter on $\mathbb{N}$. A resulting nonstandard set ${ }^{*} \mathbb{R}$ may be shown to be a linearly ordered field.

Let $S$ denote the real numbers or a set of functions such as the space of locally integrable functions, $\mathbb{L}_{\text {loc }}(\mathbb{R})$. Let $S^{\mathbb{R}+}$ be the set of nets $\left(f_{\epsilon}\right)$ in $S$ with parameter $\epsilon \in \mathbb{R}$. The nonstandard extension of $S$ will then be defined to be the quotient $\operatorname{space}^{*} S \equiv S^{\mathbb{R}+} / \sim$ for a fixed equivalence relation. The use of nets provides a generalization of the definition of Equation (12). Details of nonstandard extensions may be found in Arkeryd et al. [6].

A nonstandard Dirac delta measure results for any standard function $g$ that satisfies

$$
\int_{-\infty}^{\infty} g(x) \mathrm{d} x=1
$$

by defining the internal function,

$$
{ }^{*} \delta(x)=\Omega g(\Omega x)
$$

where $\Omega$ is any infinite hyperreal number, Laugwitz [7]. To see how the internal function (14) acts like the delta measure, perform the integration

$$
\int_{-\infty}^{\infty} \Omega g(\Omega x)^{*} \phi(x) \mathrm{d} x \approx{ }^{*} \phi(0) \int_{-\epsilon}^{\epsilon} \Omega g(\Omega x) \mathrm{d} x \approx \phi(0),
$$


where $\epsilon=\epsilon(\Omega)$ is an infinitesimal constant depending on $\Omega$, and $\phi \in D(\mathbb{R})$ is an arbitrary standard test function. The result follows by taking the standard part of Equation (15).

In standard constructions, the delta measure results from abstract limits of regularizing sequences; on the other hand, in nonstandard constructions, the functional whose standard part produces the same result as the delta measure can be represented by uncountably many internal hyperreal functions with distinct microstructures. These internal functions are called predistributions and are defined here for the space of locally integrable functions ${ }^{*} \mathbb{L}_{l o c}(\mathbb{R})$. A function $f \in{ }^{*} \mathbb{L}_{l o c}(\mathbb{R})$ is a called a predistribution if for each $n \in \mathbb{N}_{0}$ (三\{0,1,2,3, . $\}$ ) it satisfies the following: i) $f^{(n)}$ is piecewise differentiable, ii) $f^{(n)} \in{ }^{*} \mathbb{L}_{l o c}(\mathbb{R})$, and iii) there exists a generalized function $T \in D^{\prime}(\mathbb{R})$ such that

$$
\int_{-\infty}^{\infty} f^{(n)}(x)^{*} \phi(x) \mathrm{d} x \approx(-1)^{n}\left\langle T, \phi^{(n)}\right\rangle
$$

for all test functions $\phi \in D(\mathbb{R})$ and all $n \in \mathbb{N}_{0}$.

As well as the delta measure, the analysis of shock wave jump conditions requires the use of nonstandard Heaviside functions. The standard Heaviside function can be represented by uncountably many functions or predistributions with distinct microstructures. To specify a nonstandard Heaviside function, let $\epsilon_{1}$ and $\epsilon_{2}$ be two infinitesimal numbers, at least one of which is not zero, $\epsilon_{1} \approx \epsilon_{2}$ and $\epsilon_{1}<\epsilon_{2}$. A nonstandard Heaviside function $H(x)$ is defined as follows

$$
{ }^{*} H(x)= \begin{cases}0 & \text { if } x \leq \epsilon_{1} \\ { }^{*} h(x) & \text { if } \epsilon_{1}<x<\epsilon_{2} . \\ 1 & \text { if } x \geq \epsilon_{2}\end{cases}
$$

In equation $(17){ }^{*} h(x)$ is assumed to be a piecewise differentiable function, which satisfies

$$
{ }^{*} h\left(\epsilon_{1}\right) \approx 0 \text { and }{ }^{*} h\left(\epsilon_{2}\right) \approx 1 .
$$

Examples of nonstandard Heaviside functions are given in Baty et al. [1, 2]; more detail about spaces of nonstandard generalized functions and their multiplication, may be found in Todorov and Vernaeve [8]. 


\subsection{Jump Functions for Magnetogasdynamics}

Along the families $(i=1,2)$ of characteristic curves, the similarity solutions $g_{i}, v_{i}, \pi_{i}$, and $\eta_{i}$ are assumed to be smooth nonstandard jump functions across a shock front

$$
\begin{aligned}
& g_{i}\left(\xi_{i}\right)=g_{0}+[g] G_{i}\left(\xi_{i}\right), \\
& v_{i}\left(\xi_{i}\right)=v_{0}+[v] K_{i}\left(\xi_{i}\right), \\
& \pi_{i}\left(\xi_{i}\right)=\pi_{0}+[\pi] L_{i}\left(\xi_{i}\right), \\
& \eta_{i}\left(\xi_{i}\right)=\eta_{0}+[\eta] H_{i}\left(\xi_{i}\right) .
\end{aligned}
$$

Here and in the following development, the ${ }^{*}$-notation is suppressed. In Equations (17), (18), (19), and (20), each Heaviside function is also assumed to have its jump contained on the same infinitesimal interval. The definition of the shock jump interval will depend on which similarity variable $\xi_{i}$ is being applied. For diverging shock waves, $\xi_{1}$, the jump interval is defined by $(1-\epsilon, 1)$, whereas for converging shock waves, $\xi_{2}$, the jump interval is defined by $(1,1+\epsilon)$. And for both families of shock waves, $\epsilon$ is an arbitrary fixed infinitesimal. The shock wave jump intervals are defined so that they are contained in the domains of the similarity variables.

On the end points of the shock wave jump intervals the nonstandard Heaviside functions are subject to zero or one boundary data. For the $\xi_{1}$ similarity variable, the boundary conditions become

$$
\left[\begin{array}{c}
G_{1}(1-\epsilon) \\
K_{1}(1-\epsilon) \\
L_{1}(1-\epsilon) \\
H_{1}(1-\epsilon)
\end{array}\right] \approx\left[\begin{array}{l}
1 \\
1 \\
1 \\
1
\end{array}\right] \text { and }\left[\begin{array}{c}
G_{1}(1) \\
K_{1}(1) \\
L_{1}(1) \\
H_{1}(1)
\end{array}\right] \approx\left[\begin{array}{l}
0 \\
0 \\
0 \\
0
\end{array}\right]
$$

and for the $\xi_{2}$ case, the boundary data are given by

$$
\left[\begin{array}{c}
G_{2}(1) \\
K_{2}(1) \\
L_{2}(1) \\
H_{2}(1)
\end{array}\right] \approx\left[\begin{array}{l}
0 \\
0 \\
0 \\
0
\end{array}\right] \text { and }\left[\begin{array}{c}
G_{2}(1+\epsilon) \\
K_{2}(1+\epsilon) \\
L_{2}(1+\epsilon) \\
H_{2}(1+\epsilon)
\end{array}\right] \approx\left[\begin{array}{l}
1 \\
1 \\
1 \\
1
\end{array}\right] .
$$

Across a radially symmetric, shock front, the density $\rho_{1}$, the velocity $u_{1}$, and the pressure $p_{1}$ are assumed to take the limiting values for a strong MGD shock

$$
\rho_{1}=\rho_{0} \frac{\gamma+1}{\gamma-1}
$$




$$
\begin{gathered}
u_{1}=\frac{2}{\gamma+1} D, \\
p_{1}=\frac{2}{\gamma+1} \rho_{0} D^{2}-\mu h_{0}^{2} \frac{2 \gamma}{(\gamma-1)^{2}}, \\
h_{1}=h_{0} \frac{\gamma+1}{\gamma-1},
\end{gathered}
$$

where $\gamma$ is the ratio of specific heats. The strong shock jump conditions are discussed in a number of references, for example see Zel'dovich and Raizer [5].

Combining the general functional form of the similarity solutions (6) and (7) with the nonstandard jump functions (17) to (20), the Heaviside boundary data (21) and (22), and the physical boundary data (23) to (26), yields the nonstandard jump functions parameters

$$
\begin{gathered}
g_{0}=1 \text { and }[g]=\frac{\gamma+1}{\gamma-1}-1, \\
v_{0}=0 \text { and }[v]=\frac{2}{\gamma+1}, \\
\pi_{0}=0 \text { and }[\pi]=\frac{2}{\gamma+1}-\frac{1}{M_{h}^{2}} \frac{2 \gamma}{(\gamma-1)^{2}},
\end{gathered}
$$

and

$$
\eta_{0}=\frac{1}{M_{h}} \text { and }[\eta]=\frac{1}{M_{h}}\left(\frac{\gamma+1}{\gamma-1}-1\right) .
$$

In Equations (29) and (30), $M_{h}$ is the magnetic Mach number, defined as the ratio of the shock velocity to the Alfvén velocity and is given by

$$
M_{h}=D /\left(\sqrt{\mu} h_{0} / \sqrt{\rho_{0}}\right),
$$

where the square root of the magnetic permeability $\mu$ is assumed to be combined with the magnetic field strength $h$ and is suppressed in the following development.

Now to determine relationships between the nonstandard Heaviside functions, $G_{i}, K_{i}, L_{i}$, and $H_{i}$, the equations of conservation of mass, momentum, and magnetic flux must be integrated in terms of the similarity variables $\xi_{i}$ 
for $i=1,2$. To this end, substituting Equations (9) and (10) into (2), (3) and (4) produces the two systems of ODEs,

$$
\left[\begin{array}{ccc}
{[v]} & 0 & 0 \\
0 & {[\pi]} & 0 \\
0 & 0 & {[\eta]}
\end{array}\right]\left[\begin{array}{c}
K_{i} \\
L_{i} \\
H_{i}
\end{array}\right]^{\prime}=\left[\begin{array}{c}
m\left(G_{i}\right) \\
n\left(G_{i}\right) \\
o\left(G_{i}\right)
\end{array}\right]
$$

where

$$
\left[\begin{array}{c}
m\left(G_{i}\right) \\
n\left(G_{i}\right) \\
o\left(G_{i}\right)
\end{array}\right]=\left[\begin{array}{c}
\xi_{i}\left(\ln g_{i}\right)^{\prime}-\left(\kappa / \xi_{i}+\left(\ln g_{i}\right)^{\prime}\right) v_{i}-\delta(i) \\
\left.-g_{i} \lambda(i) v_{i}+\left(v_{i}-\xi_{i}\right) v_{i}{ }^{\prime}\right)-\eta_{i} \eta_{i}{ }^{\prime}-\eta_{i}{ }^{2} / \xi_{i} \\
-\eta_{i}\left(v_{i}{ }^{\prime}+(\kappa-1) v_{i} / \xi_{i}+\delta(i)+\lambda(i)\right) /\left(v_{i}-\xi_{i}\right)
\end{array}\right]
$$

and where $(\cdot)^{\prime} \equiv d / d \xi_{i}$. In Equation (34),

$$
\lambda(i) \equiv(\alpha(i)-1) / \alpha(i)
$$

Details of the derivation of Equations (34) and (35) are given in Chapter XII of Zel'dovich and Razier [5]. The parameters $\delta(i)$ and $\alpha(i)$ are assumed to be constants that depend on the initial density $\rho_{0}\left(R_{i}\right)$ and radial $R_{i}$ functions, respectively. The parameters $\alpha(i)$ are assumed to be a constant $\neq 1$. For $\alpha(i)=1$, a similarity variable occurs that has an exponential rather than a power-law character. All the shock wave jump conditions considered here are assumed to follow a power-law scaling relation.

The first equation of (34) may be integrated to solve for either $g_{i}$ or $v_{i}$. Because this equation forms a linear ODE for $v_{i}$ in terms $g_{i}$,

$$
\frac{d v_{i}}{d \xi_{i}}+\left(\frac{\kappa}{\xi_{i}}+\left(\ln g_{i}\right)^{\prime}\right) v_{i}=\xi_{i}\left(\ln g_{i}\right)^{\prime}-\delta(i),
$$

$v_{i}$ is determined as a function $g_{i}$.

Integrating Equation (37) with respect to $\xi_{i}$ yields

$$
v_{i}\left(\xi_{i}\right)=\frac{1}{\xi_{i}{ }^{\kappa} g_{i}\left(\xi_{i}\right)}\left[\xi_{i}{ }^{\kappa+1} g\left(\xi_{i}\right)-1-(\kappa+1+\delta(i)) \int_{\xi_{0}}^{\xi_{i}} \tau^{\kappa} g_{i}(\tau) d \tau\right]
$$

where $\xi_{0}=1+(i-2) \epsilon$, and $\xi_{R}=1+(i-1) \epsilon$.

To show that Equation (38) gives a jump function, it must be shown to satisfy the boundary conditions of Equation (21) (or (22)) and Equation (28). From experimental shock data, $g_{i}$ is assumed to be strictly monotonically 
increasing as a function of $\xi_{i}$; with this assumption, the integral in Equation (38) satisfies

$$
0<\int_{\xi_{0}}^{\xi_{R}} \tau^{\kappa} g_{i}(\tau) d \tau \leq g_{i}\left(\xi_{R}\right) \int_{\xi_{0}}^{\xi_{R}} \tau^{\kappa} d \tau
$$

But recalling that

$$
(1 \pm \epsilon)^{k} \approx 1 \text { for }|k|>0
$$

implies that

$$
0<\int_{\xi_{0}}^{\xi_{R}} \tau^{\kappa} g_{i}(\tau) d \tau \leq \omega
$$

where $\omega$ is an infinitesimal. Equations (38) and (41) then yield

$$
v_{i}\left(\xi_{i}\right) \approx \frac{1}{\xi_{i}{ }^{\kappa} g_{i}\left(\xi_{i}\right)}\left[\xi_{i}{ }^{\kappa+1} g_{i}\left(\xi_{i}\right)-1\right]
$$

and

$$
v_{i}\left(\xi_{R}\right) \approx \frac{2}{\gamma+1}
$$

therefore together with $v\left(\xi_{0}\right) \approx 0$, it follows from Equation (18) that $v_{i}$ is a nonstandard jump function. Equation (42) is the homogeneous part of the solution of Equation (37), which implies that the nonhomogeneous term generates an infinitesimal contribution to the velocity jump function across the shock wave.

Next, the second equation in (34) gives a linear ODE for $\pi_{i}$ in terms of $v_{i}, g_{i}$, and $\eta_{i}$

$$
\frac{d \pi_{i}}{d \xi_{i}}=-g_{i}\left(\lambda(i) v_{i}+\left(v_{i}-\xi_{i}\right) v_{i}^{\prime}\right)-\eta_{i} \eta_{i}^{\prime}-\frac{\eta_{i}^{2}}{\xi_{i}}
$$

Integrating Equation (44) with respect to $\xi_{i}$ produces

$$
\begin{aligned}
\pi_{i}\left(\xi_{i}\right)=-\lambda(i) \int_{\xi_{0}}^{\xi_{i}} g_{i}(\tau) v_{i}(\tau) d \tau-\int_{\xi_{0}}^{\xi_{i}} g_{i}(\tau)\left(v_{i}(\tau)-\tau\right) v_{i}{ }^{\prime}(\tau) d \tau \\
-\int_{\xi_{0}}^{\xi_{i}} \eta_{i}(\tau)\left(\eta_{i}{ }^{\prime}(\tau)+\eta_{i}(\tau) \tau^{-1}\right) d \tau
\end{aligned}
$$

Equation (45) may be determined as a function of $g_{i}$ and $\eta_{i}$ by combining (45) with Equation (38). Moreover, it may be shown that Equation (45) 
yields a jump function and satisfies Equation (21) (or (22)) and Equation (33). This follows by combining Equation (45) with Equation (41) and using the logic of Equations (37) to (39) to neglect all solution terms bounded by infinitesimals. Equation (45) then reduces to

$$
\pi_{i}\left(\xi_{i}\right) \approx \xi_{i}^{-\kappa} v_{i}\left(\xi_{i}\right)-v_{i}\left(\xi_{0}\right)+\frac{1}{2} \eta_{i}^{2}\left(\xi_{0}\right)-\frac{1}{2} \eta_{i}^{2}\left(\xi_{i}\right)
$$

Therefore, evaluating Equation (46) at $\xi_{0}$ and $\xi_{R}$ gives

$$
\pi_{i}\left(\xi_{0}\right) \approx 0 \text { and } \pi_{i}\left(\xi_{R}\right) \approx \frac{2}{\gamma+1}-\frac{1}{M_{h}^{2}} \frac{2 \gamma}{(\gamma-1)^{2}}
$$

and $\pi_{i}$ is a nonstandard jump function.

The third equation in (34) gives a linear ODE for $\eta_{i}$ in terms of $v_{i}$

$$
\frac{1}{\eta_{i}} \frac{d \eta_{i}}{d \xi_{i}}=-\left(v_{i}{ }^{\prime}+(\kappa-1) v_{i} / \xi_{i}+\delta(i)+\lambda(i)\right) /\left(v_{i}-\xi_{i}\right)
$$

Integrating Equation (48) with respect to the variable $\xi_{i}$ gives

$$
\eta_{i}\left(\xi_{i}\right)=\frac{1}{M_{h}} \exp \left(F_{i}\left(\xi_{i}\right)\right)
$$

where

$$
F_{i}\left(\xi_{i}\right)=-\int_{\xi_{0}}^{\xi_{i}}\left[\left[v_{i}^{\prime}(\tau)+(\kappa-1) \frac{v_{i}(\tau)}{\tau}+\delta(i)+\lambda(i)\right] /\left(v_{i}(\tau)-\tau\right)\right] d \tau
$$

By combining Equation (50) with (42) and by again assuming that $g_{i}$ is strictly monotonically increasing and that $v_{i}$ is bounded across a shock wave, Equation (50) simplifies to

$$
F_{i}\left(\xi_{i}\right) \approx \ln \left(g_{i}\left(\xi_{i}\right) / g_{i}\left(\xi_{0}\right)\right)
$$

Equation (51) results by neglecting all the solution terms of Equation (50) that are bounded by infinitesimals. It then follows that $\eta_{i}$ is a nonstandard jump function, because at $\xi_{0}$ and $\xi_{1}$, Equations (49) and (51) yield

$$
\eta_{i}\left(\xi_{0}\right) \approx \frac{1}{M_{h}} \text { and } \eta_{i}\left(\xi_{R}\right) \approx \frac{1}{M_{h}} \frac{\gamma+1}{\gamma-1}
$$


Equations (17) to (20), and Equations (23) to (26) imply that the predistributions of the Heaviside functions $G_{i}, K_{i}, L_{i}$, and $H_{i}$ modeling the microstructures of radially symmetric, diverging and converging, MGD shocks are distinct:

$$
G_{i} \neq K_{i} \neq L_{i} \neq H_{i}
$$

and

$$
G_{i} \approx H_{i}
$$

Relations (53) and (54) hold for both similarity variables $\xi_{1}$ and $\xi_{2}$ defined by Equations (6), (7), (9), and (10). Moreover, the jump functions for the density $g_{i}$, velocity $v_{i}$, pressure $\pi_{i}$, and magnetic field $\eta_{i}$ are related by the integral equations

$$
\begin{gathered}
v_{i}\left(\xi_{i}\right)=\frac{1}{\xi_{i}{ }^{\kappa} g_{i}\left(\xi_{i}\right)}\left[\xi_{i}{ }^{\kappa+1} g_{i}\left(\xi_{i}\right)-1-(\kappa+1+\delta(i)) \int_{\xi_{0}}^{\xi_{i}} \tau^{\kappa} g_{i}(\tau) d \tau\right] \\
\pi_{i}\left(\xi_{i}\right)=-\lambda(i) \int_{\xi_{0}}^{\xi_{i}} g_{i}(\tau) v_{i}(\tau) d \tau-\int_{\xi_{0}}^{\xi_{i}} g_{i}(\tau)\left(v_{i}(\tau)-\tau\right) v_{i}{ }^{\prime}(\tau) d \tau \\
-\int_{\xi_{0}}^{\xi_{i}} \eta_{i}(\tau)\left(\eta_{i}{ }^{\prime}(\tau)+\eta_{i}(\tau) \tau^{-1}\right) d \tau,
\end{gathered}
$$

and

$$
\eta_{i}\left(\xi_{i}\right)=\frac{1}{M_{h}} \exp \left(F_{i}\left(\xi_{i}\right)\right)
$$

where

$$
F_{i}\left(\xi_{i}\right)=-\int_{\xi_{0}}^{\xi_{i}}\left[\left[v_{i}{ }^{\prime}(\tau)+(\kappa-1) \frac{v_{i}(\tau)}{\tau}+\delta(i)+\lambda(i)\right] /\left(v_{i}(\tau)-\tau\right)\right] d \tau
$$

The nonstandard functions $g_{i}, v_{i}, \pi_{i}, \eta_{i} \in{ }^{*} \mathbb{L}_{l o c}(\mathbb{R})$ are solutions of the boundary value problem defined by Equations (34) and (35) and Equations (23) through $(28)$ if the equality relation $=$ is replaced with the infinitesimal equivalence relation $\approx$ in Equation (34). Equations (42), (46), (49), and (51), which neglect infinitesimal terms in the nonstandard jump functions $v_{i}, \pi_{i}$, or $\eta_{i}$, are infinitesimally close to the same flow variable jump function $\left(v_{i}, \pi_{i}\right.$, or $\left.\eta_{i}\right)$ for all radially symmetric, diverging and converging, MGD shocks for a fixed similarity variable $\xi_{i}$. Moreover, if the two similarity variables $\xi_{1}$ and $\xi_{2}$ are related by $\xi_{2}=2-\xi_{1}$ and $g_{1}\left(\xi_{1}\right)=g_{2}\left(2-\xi_{1}\right)$, 
then Equations (55), (56), (57), and (58) represent the microstructures of all radially symmetric, one-dimensional magnetogasdynamic shock waves up to the nonstandard equivalence relation $\approx$. Moreover, taking the standard part of the nonstandard jump functions $g_{i}, v_{i}, \pi_{i}$, or $\eta_{i}$ yields standard $\mathbb{L}_{\text {loc }}(\mathbb{R})$ generalized solutions for the shock wave jump conditions.

Examples of the microstructures for converging shock waves and diverging shock waves on the surface of a star are given in Baty et al. [1, 2] for the density jump function $g$,

$$
g(\xi)=1+[g]\left(\frac{\xi-1}{\epsilon}\right)^{n}
$$

for specified values of $n$. Applying a density jump function of this form to radially symmetric, diverging $(i=1)$, and converging $(i=2)$ shock waves, then yields

$$
g_{1}\left(\xi_{1}\right)=1+[g]\left(\frac{1-\xi_{1}}{\epsilon}\right)^{n}
$$

and

$$
g_{2}\left(\xi_{2}\right)=1+[g]\left(\frac{\xi_{2}-1}{\epsilon}\right)^{n} .
$$

From Equations (60) and (61) it follows that

$$
g_{1}\left(\xi_{1}\right)=g_{2}\left(2-\xi_{1}\right)
$$

so that the jump functions $v_{i}, \pi_{i}$, and $\eta_{i}$ satisfy

$$
\begin{aligned}
& v_{1}\left(\xi_{1}\right) \approx v_{2}\left(2-\xi_{1}\right), \\
& \pi_{1}\left(\xi_{1}\right) \approx \pi_{2}\left(2-\xi_{1}\right),
\end{aligned}
$$

and

$$
\eta_{1}\left(\xi_{1}\right) \approx \eta_{2}\left(2-\xi_{1}\right)
$$

on the interval $(1-\epsilon, 1)$.

\section{Summary and Conclusions}

This article derived nonstandard jump conditions for radially symmetric, onedimensional, diverging and converging shock waves in magnetogasdynamic flows. Predistributions of the Heaviside function were applied to model the 
microstructure of the flow field across shock waves. For flow variables with their jumps defined on the same infinitesimal interval, it was shown that the equations of motion written in nonconservative form produced unambiguous relations between the various nonstandard Heaviside functions. Because inviscid flow was assumed, the governing equations were underdetermined and only produced the relations between the field variable Heaviside functions. The main conclusions of the study are as follows:

1. Nonstandard analysis was used to construct generalized solutions of the radially symmetric, one-dimensional magnetogasdynamic equations across shock waves. The functions constructed here are piecewise differentiable elements of the function space ${ }^{*} \mathbb{L}_{l o c}(\mathbb{R})$. The relations between the nonstandard Heaviside functions were derived unambiguously from the equations of motion in nonconservative form. Distinct nonstandard jump functions were obtained for the density, velocity, pressure, and magnetic field for both diverging and converging flows.

2. If the two similarity variables $\xi_{1}$ and $\xi_{2}$ are related affinely and orientated on a common shock jump interval for a specified density jump function, the microstructures of all radially symmetric, one-dimensional magnetogasdynamic shock waves coincide up to the nonstandard equivalence relation $\approx$.

\section{References}

[1] Baty, R. S., Farassat, F., and Tucker, D. H., "Nonstandard Analysis and Shock Wave Jump Conditions for Converging Shock Waves," Journal of Mathematical Physics, 49, No. 6 pp. 063101 1-18, 2008.

[2] Baty, R. S., and Tucker, D. H., Hyperreal Jump Conditions for Shock Waves on the Surface of a Star, LA-14364, Los Alamos National Laboratory, May 2008, Electronic copy available: http://www.doe.gov/bridge.

[3] Christer, A. H., and Helliwell, J. B., "Cylindrical Shock and Detonation Waves in Magnetogasdynamics," Journal of Fluid Mechanics, 39, Part 4, pp. 705-725, 1969.

[4] Srivastava, R. C., "The Emergence of a Spherical Magnetogasdynamic Shock Wave at the Surface of a Star," Astrophysics and Space Science, 92, pp. 359-364, 1983. 
[5] Zel'dovich, Ya. B., and Raizer, Yu. P., Physics of Shock Waves and HighTemperature Hydrodynamic Phenomena, Academic Press, 1966 (Volume I) 1967 (Volume II).

[6] Arkeryd, L. O., Cutland, N. J., and Henson, C. W., Nonstandard Analysis Theory and Application, Kluwer Academic Publishers, 1997.

[7] Laugwitz, D., The Theory of Infinitesimals An Introduction to Nonstandard Analysis, Accademia Nazionale Dei Lincei, 1980.

[8] Todorov, T. D., and Vernaeve, H. Full Algebra of Generalized Functions and Non-Standard Asymptotic Analysis, Unpublished Manuscript, arXiv:0712.2603v1, 2007. 
This page left blank intentionally. 
This page left blank intentionally. 
This page left blank intentionally. 
This report has been reproduced directly from the best available copy. It is available electronically on the Web (http://www.doe.gov/bridge).

Copies are available for sale to U.S. Department of Energy employees and contractors from:

Office of Scientific and Technical Information

P.O. Box 62

Oak Ridge, TN 37831

(865) 576-8401

Copies are available for sale to the public from: National Technical Information Service

U.S. Department of Commerce

5285 Port Royal Road

Springfield, VA 22161

(800) 553-6847 
- Los Alamos NATIONAL LABORATORY

EST.1943 